\title{
New multipled common fixed point theorems in Menger PM-spaces
}

\author{
Xiaohuan Mu, Chuanxi Zhu* and Zhaoqi Wu
}

${ }^{\text {*Correspondence: }}$

chuanxizhu@126.com

Department of Mathematics,

Nanchang University, Nanchang,

330031, P.R. China

\begin{abstract}
In this work, we introduce a new $\varphi$-contractive mapping; following that, we obtain some multipled common fixed point theorems for a pair of mappings

$T: \underbrace{X \times X \times \cdots \times X}_{m \text {-times }} \rightarrow X$ and $A: X \rightarrow X$. The main results of this paper are generalization of the main results of Kutbi et al. (Fixed Point Theory Appl. 2015(1):32, 2015). As an illustration, we give an example to demonstrate the validity of the obtained results.
\end{abstract}

MSC: Primary 47H10; secondary 46S10

Keywords: multipled common fixed point; Menger PM-spaces; $\varphi$-contractive mapping

\section{Introduction}

In 1942, Menger [1] initiated the study of probabilistic metric spaces. Since then, many scholars have studied the existence of fixed points or solutions of nonlinear equations under various types of conditions in Menger spaces (see [2-8]). Precisely, Sehgal and Bharucha-Reid [9] introduced probabilistic $q$-contractions and proved corresponding unique fixed point results by giving a generalization of the classical Banach fixed point principle. Then, we point out an important theoretical development in the way of defining the concept of contractive mapping in Menger spaces. In 1984, Khan et al. [10] introduced the concept of altering distance function. Choudhury and Das [11] defined a generalized contractive condition with the help of such functions and established an unique fixed point result. In 2010, Jachymski [12] established a fixed point theorem for probabilistic $\varphi$-contractions. Dutta et al. [13] defined nonlinear generalized contractive type mappings in Menger PM-spaces and proved their theorems under the mapping in G-complete Menger PM-spaces. Recently, [14-18] have studied some new fixed point theorems in Menger PM-spaces and fuzzy metric spaces.

Coupled and tripled fixed point results were studied in [19-21]. In this paper, from the idea of $\psi$-contractive type mappings in [16], we introduce a new $\varphi$-contractive mapping. Following this, we obtain some multipled common fixed point theorems for a pair of mappings $T: \underbrace{X \times X \times \cdots \times X}_{m \text {-times }} \rightarrow X$ and $A: X \rightarrow X$, which is a generalization of [16]. As an illustration, we give an example to demonstrate the validity of the obtained results.

(c) $2015 \mathrm{Mu}$ et al. This article is distributed under the terms of the Creative Commons Attribution 4.0 International License (http://creativecommons.org/licenses/by/4.0/), which permits unrestricted use, distribution, and reproduction in any medium, provided you give appropriate credit to the original author(s) and the source, provide a link to the Creative Commons license, and indicate if changes were made. 


\section{Preliminaries}

Let $\mathbb{R}$ denote the set of reals, $\mathbb{R}^{+}$the nonnegative reals and $\mathbb{Z}^{+}$be the set of all positive integers. A mapping $F: \mathbb{R} \rightarrow \mathbb{R}^{+}$is called a distribution function if it is nondecreasing and left-continuous with $\inf _{t \in \mathbb{R}} F(t)=0$ and $\sup _{t \in \mathbb{R}} F(t)=1$. We will denote by $\mathscr{D}$ the set of all distribution functions, while $H$ will always denote the specific distribution function defined by

$$
H(t)= \begin{cases}0, & t \leq 0 \\ 1, & t>0\end{cases}
$$

A mapping $\Delta:[0,1] \times[0,1] \rightarrow[0,1]$ is called a triangular norm (for short, a $t$-norm) if the following conditions are satisfied: $\Delta(a, 1)=a ; \Delta(a, b)=\Delta(b, a) ; a \geq b, c \geq d \Rightarrow$ $\Delta(a, c) \geq \Delta(b, d) ; \Delta(a, \Delta(b, c))=\Delta(\Delta(a, b), c)$.

Three examples of $t$-norm are $\Delta_{m}(a, b)=\min \{a, b\}, \Delta_{p}(a, b)=a b$ and $\Delta_{L}=\max \{a+b-$ $1,0\}$, these $t$-norms are related in the following way: $\Delta_{L} \leq \Delta_{p} \leq \Delta_{m}$.

Definition 2.1 [22] A Menger PM-space is a triple $(X, F, \Delta)$ where $X$ is a nonempty set, $\Delta$ is a continuous $t$-norm and $F$ is a mapping from $X \times X$ into $\mathscr{D}^{+}$such that, if $F_{x, y}$ denotes the value of $F$ at the pair $(x, y)$, the following conditions hold:

$(\mathrm{PM}-1) F_{x, y}(t)=H(t)$ if and only if $x=y, t>0$;

(PM-2) $F_{x, y}=F_{y, x}$ for all $x, y \in X$;

(PM-3) $F_{x, y}(t+s) \geq \Delta\left(F_{x, z}(t), F_{z, y}(s)\right)$ for all $x, y, z \in X$ and $s, t \geq 0$.

Definition 2.2 [23] Let $(X, F, \Delta)$ be a Menger PM-space. Then

(i) a sequence $\left\{x_{n}\right\}$ is said to be convergent to $x \in X$ if for every $\epsilon>0$ and $\lambda>0$, there exists a positive integer $Z^{+}$such that $F_{x_{n}, x}(\epsilon)>1-\lambda$ whenever $n \geq Z^{+}$;

(ii) a sequence $\left\{x_{n}\right\}$ in $X$ is called a Cauchy sequence if for every $\epsilon>0$ and $\lambda>0$, there exists a positive integer $Z^{+}$such that $F_{x_{n}, x_{m}}(\epsilon)>1-\lambda$ whenever $n, m \geq Z^{+}$;

(iii) a Menger PM-space is said to be $M$-complete if every Cauchy sequence in $X$ is convergent to a point in $X$;

(iv) a sequence $\left\{x_{n}\right\}$ is said to be a G-Cauchy sequence if $\lim _{n \rightarrow \infty} F_{x_{n}, x_{n+m}}(t)=1$ for each $m \in Z^{+}$and $t>0$

(v) the space $(X, F, \Delta)$ is called $G$-complete if every $G$-Cauchy sequence in $X$ is convergent to a point in $X$.

According to [2], the $(\epsilon, \lambda)$-topology in a Menger PM-space $(X, F, \Delta)$ is introduced by the family of neighborhoods $N_{x}$ of a point $x \in X$ given by $N_{x}=\left\{N_{x}(\epsilon, \lambda): \epsilon>0, \lambda \in(0,1)\right\}$, where $N_{x}(\epsilon, \lambda)=\left\{y \in X: F_{x, y}(\epsilon)>1-\lambda\right\}$. Then $(\epsilon, \lambda)$-topology is a Hausdorff topology.

Definition 2.3 [11] A function $\phi: R^{+} \rightarrow R^{+}$is said to be a $\phi$-function if it satisfies the following conditions:

(i) $\phi(t)=0$ if and only if $t=0$;

(ii) $\phi(t)$ is strictly increasing and $\phi(t) \rightarrow \infty$ as $t \rightarrow \infty$;

(iii) $\phi$ is left-continuous in $(0, \infty)$;

(iv) $\phi$ is continuous at 0 .

Definition 2.4 Let $X$ be a nonempty set. Let $T: \underbrace{X \times X \times \cdots \times X}_{m \text {-times }} \rightarrow X$ and $A: X \rightarrow X$ be two mappings. $A$ is said to be commutative with $T$ if $A T(x, y, \ldots, z)=T(A x, A y, \ldots, A z)$ for 
all $x, y, \ldots, z \in X$. A point $u \in X$ is called a multipled common fixed point of $T$ and $A$ if $u=A u=T(u, u, \ldots, u)$.

\section{Main results}

In this section, we denote by $\Phi$ the class of all nondecreasing functions $\varphi: R^{+} \rightarrow R^{+}$such that $\varphi$ is continuous at $0, \varphi(0)=0$ and $\varphi^{n}\left(a_{n}\right) \rightarrow 0$ whenever $a_{n} \rightarrow 0$ as $n \rightarrow \infty$.

Theorem 3.1 Let $(X, F, \Delta)$ be a G-complete Menger space with $\Delta$ a continuous $t$-norm. Let $T: \underbrace{X \times X \times \cdots \times X}_{m \text {-times }} \rightarrow X$ and $A: X \rightarrow X$ be two mappings satisfying the following inequality:

$$
\begin{aligned}
& \frac{1}{F_{T(x, y, \ldots, z), T(p, q, \ldots, r)}(\phi(c t))}-1 \\
& \quad \leq \varphi\left\{\frac{\left(\frac{1}{F_{A x, A p}(\phi(t))}-1\right)+\left(\frac{1}{F_{A y, A q}(\phi(t))}-1\right)+\cdots+\left(\frac{1}{F_{A z, A r}(\phi(t))}-1\right)}{m}\right\}
\end{aligned}
$$

for all $x, y, \ldots, z, p, q, \ldots, r \in X, c \in(0,1), \varphi \in \Phi, \phi$ is a $\phi$-function, $t>0$, such that $F_{A x, A p}(\phi(t))>0, F_{A y, A p}(\phi(t))>0, \ldots, F_{A z, A r}(\phi(t))>0$, where $T(X \times X \times \cdots \times X) \subset A(X), A$ is continuous and commutative with $T$. Then there exists a unique multipled common fixed point of $A$ and $T$, i.e., there exists a unique $u \in X$ such that $u=A u=T(u, u, \ldots, u)$.

Proof Let $\left\{x_{n}\right\}_{n=1}^{\infty},\left\{y_{n}\right\}_{n=1}^{\infty}, \ldots,\left\{z_{n}\right\}_{n=1}^{\infty}$ be $m$-times sequences in $X$ such that $A x_{n+1}=T\left(x_{n}, y_{n}\right.$, $\left.\ldots, z_{n}\right)$ and $A y_{n+1}=T\left(y_{n}, \ldots, z_{n}, x_{n}\right), A z_{n+1}=T\left(z_{n}, x_{n}, y_{n}, \ldots\right)$. From $\sup _{t \in R} F_{A x_{0}, A x_{1}}(t)=$ $1, \sup _{t \in R} F_{A y_{0}, A y_{1}}(t)=1, \ldots, \sup _{t \in R} F_{A z_{0}, A z_{1}}(t)=1$ and the definition of $\phi$, one can find $t>0$ such that $F_{A x_{0}, A x_{1}}\left(\phi\left(\frac{t}{c}\right)\right)>0, F_{A y_{0}, A y_{1}}\left(\phi\left(\frac{t}{c}\right)\right)>0, \ldots, F_{A z_{0}, A z_{1}}\left(\phi\left(\frac{t}{c}\right)\right)>0$. From (3.1), we have

$$
\begin{aligned}
& \frac{1}{F_{A x_{1}, A x_{2}}(\phi(t))}-1 \\
& \quad=\frac{1}{F_{T\left(x_{0}, y_{0}, \ldots, z_{0}\right), T\left(x_{1}, y_{1}, \ldots, z_{1}\right)}(\phi(t))}-1 \\
& \quad \leq \varphi\left\{\frac{\left(\frac{1}{F_{A x_{0}, A x_{1}}\left(\phi\left(\frac{t}{c}\right)\right)}-1\right)+\left(\frac{1}{F_{A y_{0}, A y_{1}}\left(\phi\left(\frac{t}{c}\right)\right)}-1\right)+\cdots+\left(\frac{1}{F_{A z_{0}, A z_{1}}\left(\phi\left(\frac{t}{c}\right)\right)}-1\right)}{m}\right\} .
\end{aligned}
$$

Similarly, we have

$$
\begin{aligned}
& \frac{1}{F_{A y_{1}, A y_{2}}(\phi(t))}-1 \\
& \quad \leq \varphi\left\{\frac{\left(\frac{1}{F_{A y_{0}, A y_{1}}\left(\phi\left(\frac{t}{c}\right)\right)}-1\right)+\cdots+\left(\frac{1}{F_{A z_{0}, A z_{1}}\left(\phi\left(\frac{t}{c}\right)\right)}-1\right)+\left(\frac{1}{m}-1\right)}{F_{A x_{0}, A x_{1}}\left(\phi\left(\frac{t}{c}\right)\right)}-1\right) \\
& \quad \ldots, \\
& \quad \frac{1}{F_{A z_{1}, A z_{2}}(\phi(t))}-1 \\
& \quad \leq \varphi\left\{\frac{\left(\frac{1}{F_{A z_{0}, A z_{1}}\left(\phi\left(\frac{t}{c}\right)\right)}-1\right)+\left(\frac{1}{F_{A x_{0}, A x_{1}}\left(\phi\left(\frac{t}{c}\right)\right)}-1\right)+\left(\frac{1}{F_{A y_{0}, A y_{1}}\left(\phi\left(\frac{t}{c}\right)\right)}-1\right)+\cdots}{m}\right\} .
\end{aligned}
$$


Suppose that $P_{0}(t)=\frac{\left(\frac{1}{F_{A x_{0}, A x_{1}}(\phi(t))}-1\right)+\left(\frac{1}{F_{A y_{0}, A y_{1}}(\phi(t))}-1\right)+\cdots+\left(\frac{1}{F_{A z_{0}, A z_{1}}(\phi(t))}-1\right)}{m}$, from (3.2), (3.3) and (3.4) we deduce that $F_{A x_{1}, A x_{2}}(\phi(t))>0, F_{A y_{1}, A y_{2}}(\phi(t))>0, \ldots, F_{A z_{1}, A z_{2}}(\phi(t))>0$, and so $F_{A x_{1}, A x_{2}}\left(\phi\left(\frac{t}{c}\right)\right)>0, F_{A y_{1}, A y_{2}}\left(\phi\left(\frac{t}{c}\right)\right)>0, \ldots, F_{A z_{1}, A z_{2}}\left(\phi\left(\frac{t}{c}\right)\right)>0$, then we have

$$
\begin{aligned}
& \frac{1}{F_{A x_{2}, A x_{3}}(\phi(t))}-1 \\
& \quad=\frac{1}{F_{T\left(x_{1}, y_{1}, \ldots, z_{1}\right), T\left(x_{2}, y_{2}, \ldots, z_{2}\right)}(\phi(t))}-1 \\
& \quad \leq \varphi\left\{\frac{\left(\frac{1}{F_{A x_{1}, A x_{2}}\left(\phi\left(\frac{t}{c}\right)\right)}-1\right)+\left(\frac{1}{F_{A y_{1}, A y_{2}}\left(\phi\left(\frac{t}{c}\right)\right)}-1\right)+\cdots+\left(\frac{1}{m}-1\right)}{m}\right\} \\
& \quad \leq \varphi\left\{\frac{\varphi\left(P_{0}\left(\frac{t}{c^{2}}\right)\right)+\varphi\left(P_{0}\left(\frac{t}{c^{2}}\right)\right)+\cdots+\varphi\left(P_{0}\left(\frac{t}{c^{2}}\right)\right)}{m}\right\}=\varphi^{2}\left\{P_{0}\left(\frac{t}{c^{2}}\right)\right\} .
\end{aligned}
$$

Similarly, we have

$$
\begin{aligned}
& \frac{1}{F_{A y_{2}, A y_{3}}(\phi(t))}-1 \leq \varphi^{2}\left\{P_{0}\left(\frac{t}{c^{2}}\right)\right\}, \\
& \ldots, \\
& \frac{1}{F_{A z_{2}, A z_{3}}(\phi(t))}-1 \leq \varphi^{2}\left\{P_{0}\left(\frac{t}{c^{2}}\right)\right\} .
\end{aligned}
$$

Repeating the above procedure, we get

$$
\frac{1}{F_{A x_{n}, A x_{n+1}}(\phi(t))}-1 \leq \varphi^{n}\left\{P_{0}\left(\frac{t}{c^{n}}\right)\right\} .
$$

If we change $A x_{0}, A y_{0}, \ldots, A z_{0}$ with $A x_{r}, A y_{r}, \ldots, A z_{r}$ in (3.5), then for all $n>r$ we get

$$
\frac{1}{F_{A x_{n}, A x_{n+1}}\left(\phi\left(c^{r} t\right)\right)}-1 \leq \varphi^{n-r}\left\{P_{r}\left(\frac{c^{r} t}{c^{n-r}}\right)\right\} .
$$

Since $\varphi^{n}\left(a_{n}\right) \rightarrow 0$ whenever $a_{n} \rightarrow 0$ as $n \rightarrow \infty$, therefore the above inequality implies that

$$
\lim _{n \rightarrow \infty} F_{A x_{n}, A x_{n+1}}\left(\phi\left(c^{r} t\right)\right)=1 .
$$

Now, let $\epsilon$ be given, using the properties of $\phi$-function, we can find $r \in Z^{+}$such that $\phi\left(c^{r} t\right)<\epsilon$. Then we have

$$
\lim _{n \rightarrow \infty} F_{A x_{n}, A x_{n+1}}(\epsilon) \geq \lim _{n \rightarrow \infty} F_{A x_{n}, A x_{n+1}}\left(\phi\left(c^{r} t\right)\right)=1 .
$$

By using a triangle inequality, we obtain

$$
F_{A x_{n}, A x_{n+p}}(\epsilon) \geq \Delta(\underbrace{F_{A x_{n}, A x_{n+1}}\left(\frac{\epsilon}{p}\right), \Delta\left(F_{A x_{n+1}, A x_{n+2}}\left(\frac{\epsilon}{p}\right), \ldots, F_{A x_{n+p-1}, A x_{n+p}}\left(\frac{\epsilon}{p}\right)\right)}_{p \text {-times }}) .
$$

Let $n \rightarrow \infty$ and make use of (3.7), for any integer $p$, we get

$$
\lim _{n \rightarrow \infty} F_{A x_{n}, A x_{n+p}}(\epsilon)=1 \quad \text { for every } \epsilon>0 .
$$


Hence $\left\{A x_{n}\right\}$ is a G-Cauchy sequence. Similarly, we can obtain that $\left\{A y_{n}\right\}, \ldots,\left\{A z_{n}\right\}$ are GCauchy sequences. Since $(X, F, \Delta)$ is $G$-complete, therefore $\lim _{n \rightarrow \infty} A x_{n}=u, \lim _{n \rightarrow \infty} A y_{n}=$ $v, \ldots, \lim _{n \rightarrow \infty} A z_{n}=w$ for some $u, v, \ldots, w \in X$.

Now we show that $A u=T(u, v, \ldots, w)$.

By the continuity of $A$, we can obtain that $\lim _{n \rightarrow \infty} A A x_{n}=A u, \lim _{n \rightarrow \infty} A A y_{n}=A v, \ldots$, $\lim _{n \rightarrow \infty} A A z_{n}=A w$. Then the commutativity of $A$ with $T$ implies that $A A x_{n+1}=T\left(A x_{n}\right.$, $\left.A y_{n}, \ldots, A z_{n}\right)$. From (3.1), we obtain

$$
\begin{aligned}
& \frac{1}{F_{A A x_{n+1}, T(u, v, \ldots, w)}(\phi(t))}-1 \\
& \quad=\frac{1}{F_{T\left(A x_{n}, A y_{n}, \ldots, A z_{n}\right), T(u, v, \ldots, w)}(\phi(t))}-1 \\
& \quad \leq \varphi\left\{\frac{\left(\frac{1}{F_{A A x_{n}, A u}\left(\phi\left(\frac{t}{c}\right)\right)}-1\right)+\left(\frac{1}{F_{A A y_{n}, A v}\left(\phi\left(\frac{t}{c}\right)\right)}-1\right)+\cdots+\left(\frac{1}{F_{A A z_{n}, A w}\left(\phi\left(\frac{t}{c}\right)\right)}-1\right)}{m}\right\} .
\end{aligned}
$$

Letting $n \rightarrow \infty$, since $\varphi(0)=0$, we have $\lim _{n \rightarrow \infty} A A x_{n+1}=T(u, v, \ldots, w)$, from the above inequality, we get $A u=T(u, v, \ldots, w)$. Similarly, we have $A v=T(v, \ldots, w, u), \ldots, A w=$ $T(w, u, v, \ldots)$.

Next we will show that $A u=u$. From (3.1), we have

$$
\begin{aligned}
& \frac{1}{F_{A x_{1}, A u}(\phi(t))}-1 \\
& =\frac{1}{F_{T\left(x_{0}, y_{0}, \ldots, z_{0}\right), T(u, v, \ldots, w)}(\phi(t))}-1 \\
& \quad \leq \varphi\left\{\frac{\left(\frac{1}{F_{A x_{0}, A u}\left(\phi\left(\frac{t}{c}\right)\right)}-1\right)+\left(\frac{1}{F_{A y_{0}, A v}\left(\phi\left(\frac{t}{c}\right)\right)}-1\right)+\cdots+\left(\frac{1}{F_{A z_{0}, A w}\left(\phi\left(\frac{t}{c}\right)\right)}-1\right)}{m}\right\}, \\
& \frac{1}{F_{A y_{1}, A v}(\phi(t))}-1 \\
& \quad \leq \varphi\left\{\frac{\left(\frac{1}{F_{A y_{0}, A v}\left(\phi\left(\frac{t}{c}\right)\right)}-1\right)+\cdots+\left(\frac{1}{F_{A z_{0}, A w}\left(\phi\left(\frac{t}{c}\right)\right)}-1\right)+\left(\frac{1}{F_{A x_{0}, A u}\left(\phi\left(\frac{t}{c}\right)\right)}-1\right)}{m}\right\} \\
& \frac{1}{F_{A z_{1}, A w}(\phi(t))}-1 \\
& \quad \leq \varphi\left\{\frac{\left(\frac{1}{F_{A z_{0}, A w}\left(\phi\left(\frac{t}{c}\right)\right)}-1\right)+\left(\frac{1}{F_{A x_{0}, A u}\left(\phi\left(\frac{t}{c}\right)\right)}-1\right)+\left(\frac{1}{F_{A y_{0}, A v}\left(\phi\left(\frac{t}{c}\right)\right)}-1\right)+\cdots}{m}\right\} .
\end{aligned}
$$

Suppose that $Q_{0}(t)=\frac{\left(\frac{1}{F_{A x_{0}, A u}(\phi(t))}-1\right)+\left(\frac{1}{F_{A y_{0}, A v}(\phi(t))}-1\right)+\cdots+\left(\frac{1}{F_{A z_{0}, A w}(\phi(t))}-1\right)}{m}$. Combining (3.8), (3.9) with (3.10), we obtain

$$
\begin{aligned}
& \frac{1}{F_{A x_{2}, A u}(\phi(t))}-1 \\
& \quad \leq \varphi\left\{\frac{\left(\frac{1}{F_{A x_{1}, A u}\left(\phi\left(\frac{t}{c}\right)\right)}-1\right)+\left(\frac{1}{F_{A y_{1}, A v}\left(\phi\left(\frac{t}{c}\right)\right)}-1\right)+\cdots+\left(\frac{1}{F_{A z_{1}, A w}\left(\phi\left(\frac{t}{c}\right)\right)}-1\right)}{m}\right\}
\end{aligned}
$$




$$
\begin{aligned}
& \leq \varphi\left\{\frac{\varphi\left(Q_{0}\left(\frac{t}{c^{2}}\right)\right)+\varphi\left(Q_{0}\left(\frac{t}{c^{2}}\right)\right)+\cdots \varphi\left(Q_{0}\left(\frac{t}{c^{2}}\right)\right)+}{m}\right\}=\varphi^{2}\left\{Q_{0}\left(\frac{t}{c^{2}}\right)\right\}, \\
& \frac{1}{F_{A y_{2}, A v}(\phi(t))}-1 \leq \varphi^{2}\left\{Q_{0}\left(\frac{t}{c^{2}}\right)\right\}, \\
& \ldots, \\
& \frac{1}{F_{A z_{2}, A w}(\phi(t))}-1 \leq \varphi^{2}\left\{Q_{0}\left(\frac{t}{c^{2}}\right)\right\} .
\end{aligned}
$$

Repeating the above procedure, we obtain

$$
\frac{1}{F_{A x_{n}, A u}(\phi(t))}-1 \leq \varphi^{n}\left\{Q_{0}\left(\frac{t}{c^{n}}\right)\right\} .
$$

Since $\varphi^{n}\left(a_{n}\right) \rightarrow 0$ whenever $a_{n} \rightarrow 0$ as $n \rightarrow \infty$, we have $\lim _{n \rightarrow \infty} A x_{n}=A u$, which implies that $A u=u=T(u, v, \ldots, w)$. Similarly, we have $A v=v=T(v, \ldots, w, u), \ldots, A w=w=$ $T(w, u, v, \ldots)$.

Finally, we show that $u=v=\cdots=w$.

For a better expression, we denote $u=e_{1}, v=e_{2}, \ldots, w=e_{m}$, then $A e_{1}=e_{1}=T\left(e_{1}, e_{2}, e_{3}\right.$, $\left.\ldots, e_{m-1}, e_{m}\right), A e_{2}=e_{2}=T\left(e_{2}, e_{3}, \ldots, e_{m-1}, e_{m}, e_{1}\right), \ldots, A e_{m}=e_{m}=T\left(e_{m}, e_{1}, e_{2}, e_{3}, \ldots, e_{m-1}\right)$.

First, we prove that $F_{e_{1}, e_{2}}(\phi(s))>0$ for all $s>0$. By the definition of $\phi$, we have $\phi\left(\frac{s}{c^{n}}\right) \rightarrow \infty$ as $n \rightarrow \infty$. Since $\sup _{n \in Z_{+}} F_{e_{1}, e_{2}}\left(\phi\left(\frac{s}{c^{n}}\right)\right)=1, \sup _{n \in Z_{+}} F_{e_{2}, e_{3}}\left(\phi\left(\frac{s}{c^{n}}\right)\right)=1, \ldots$, $\sup _{n \in Z_{+}} F_{e_{m}, e_{1}}\left(\phi\left(\frac{s}{c^{n}}\right)\right)=1$, we deduce that there exists $n \in Z^{+}$such that $F_{e_{1}, e_{2}}\left(\phi\left(\frac{s}{c^{n}}\right)\right)>$ $0, F_{e_{2}, e_{3}}\left(\phi\left(\frac{s}{c^{n}}\right)\right)>0, \ldots, F_{e_{m}, e_{1}}\left(\phi\left(\frac{s}{c^{n}}\right)\right)>0$. Using (3.1), we obtain

$$
\begin{aligned}
& \frac{1}{F_{e_{1}, e_{2}}\left(\phi\left(\frac{s}{c^{n-1}}\right)\right)}-1 \\
& \quad=\frac{1}{F_{T\left(e_{1}, e_{2}, \ldots, e_{m-1}, e_{m}\right), T\left(e_{2}, \ldots, e_{m-1}, e_{m}, e_{1}\right)}\left(\phi\left(\frac{s}{c^{n-1}}\right)\right)}-1 \\
& \quad \leq \varphi\left\{\frac{\left(\frac{1}{F_{e_{1}, e_{2}}\left(\phi\left(\frac{s}{c^{n}}\right)\right)}-1\right)+\cdots+\left(\frac{1}{F_{e_{m-1}, e_{m}}\left(\phi\left(\frac{s}{c^{n}}\right)\right)}-1\right)+\left(\frac{1}{m}-1\right)}{m}\right\},
\end{aligned}
$$

which implies that $F_{e_{1}, e_{2}}\left(\phi\left(\frac{s}{c^{n-1}}\right)\right)>0$. Similarly, we can obtain that $F_{e_{2}, e_{3}}\left(\phi\left(\frac{s}{c^{n-1}}\right)\right)>0, \ldots$, $F_{e_{m}, e_{1}}\left(\phi\left(\frac{s}{c^{n-1}}\right)\right)>0$. By repeating a similar reasoning $n$-times, we deduce that $F_{e_{1}, e_{2}}(\phi(s))>$ $0, F_{e_{2}, e_{3}}(\phi(s))>0, \ldots, F_{e_{m}, e_{1}}(\phi(s))>0$ for all $s>0$.

Second, we show that $F_{e_{1}, e_{2}}(\phi(s))=1$. In fact, for every $s>0$, we have $F_{e_{1}, e_{2}}\left(\phi\left(\frac{s}{c^{i}}\right)\right)>0$ for all $1 \leq i \leq n$ and $n \in Z^{+}$. Then, by using (3.1), we get

$$
\begin{aligned}
& \frac{1}{F_{e_{1}, e_{2}}(\phi(s))}-1 \\
& =\frac{1}{F_{T\left(e_{1}, e_{2}, \ldots, e_{m-1}, e_{m}\right), T\left(e_{2}, \ldots, e_{m-1}, e_{m}, e_{1}\right)}(\phi(s))}-1 \\
& \leq \varphi\left\{\frac{\left(\frac{1}{F_{e_{1}, e_{2}}\left(\phi\left(\frac{s}{c}\right)\right)}-1\right)+\cdots+\left(\frac{1}{F_{e_{m-1}, e_{m}}\left(\phi\left(\frac{s}{c}\right)\right)}-1\right)+\left(\frac{1}{F_{e_{m}, e_{1}}\left(\phi\left(\frac{s}{c}\right)\right)}-1\right)}{m}\right\}, \\
& \frac{1}{F_{e_{2}, e_{3}}(\phi(s))}-1 \\
& =\frac{1}{F_{T\left(e_{2}, e_{3}, \ldots, e_{m}, e_{1}\right), T\left(e_{3}, e_{4}, \ldots, e_{1}, e_{2}\right)}(\phi(s))}-1
\end{aligned}
$$




$$
\begin{aligned}
& \quad \leq \varphi\left\{\frac{\left(\frac{1}{F_{e_{2}, e_{3}}\left(\phi\left(\frac{s}{c}\right)\right)}-1\right)+\cdots+\left(\frac{1}{F_{e_{m}, e_{1}}\left(\phi\left(\frac{s}{c}\right)\right)}-1\right)+\left(\frac{1}{m}-1\right)}{F_{e_{1}, e_{2}}\left(\phi\left(\frac{s}{c}\right)\right)}-1\right) \\
& \ldots, \\
& \frac{1}{F_{e_{m}, e_{1}}(\phi(s))}-1 \\
& \quad=\frac{1}{F_{T\left(e_{m}, e_{1}, \ldots, e_{m-2}, e_{m-1}\right), T\left(e_{1}, e_{2}, \ldots, e_{m-1}, e_{m}\right)}(\phi(s))}-1 \\
& \quad \leq \varphi\left\{\frac{\left(\frac{1}{F_{e_{m}, e_{1}}\left(\phi\left(\frac{s}{c}\right)\right)}-1\right)+\left(\frac{1}{F_{e_{1}, e_{2}}\left(\phi\left(\frac{s}{c}\right)\right)}-1\right)+\cdots+\left(\frac{1}{F_{e_{m-1}, e_{m}}\left(\phi\left(\frac{s}{c}\right)\right)}-1\right)}{m}\right\} .
\end{aligned}
$$

Suppose that $E(s)=\frac{\left(\frac{1}{F_{e_{1}, e_{2}}(\phi(s)}-1\right)+\left(\frac{1}{F_{e_{2}, e_{3}(\phi(s))}}-1\right)+\cdots+\left(\frac{1}{F_{e_{m}, e_{1}}(\phi(s)}\right)^{-1)}}{m}$, then $E(s) \leq \varphi\left\{E\left(\frac{s}{c}\right)\right\}$. By $n$-iterations we get

$$
\frac{1}{F_{e_{1}, e_{2}}(\phi(s))}-1 \leq \varphi\left\{E\left(\frac{s}{c}\right)\right\} \leq \varphi^{2}\left\{E\left(\frac{s}{c^{2}}\right)\right\} \leq \cdots \leq \varphi^{n}\left\{E\left(\frac{s}{c^{n}}\right)\right\} .
$$

Thus, since $\varphi^{n}\left(a_{n}\right) \rightarrow 0$ whenever $a_{n} \rightarrow 0$ as $n \rightarrow \infty$, we get $F_{e_{1}, e_{2}}(\phi(s))=1$. It follows that $F_{e_{1}, e_{2}}(t)=H(t)$ for all $t>0$. In fact, if $t$ is not in range of $\phi$, since $\phi$ is continuous at 0 , there exists $s>0$ such that $\phi(s)<t$. This implies that $F_{e_{1}, e_{2}}(t) \geq F_{e_{1}, e_{2}}(\phi(s))=1$, then $e_{1}=e_{2}$. Similarly, we have $e_{2}=e_{3}, \ldots, e_{m}=e_{1}$, i.e., $u=v=\cdots=w$. Thus, $u \in X$ is the unique multipled common fixed point of $A$ and $T$.

Taking $m=1$ in Theorem 3.1, then $T: X \rightarrow X, A=I_{x}$ (the identity mapping on $X$ ). It is obvious that $T(X) \subset A(X), A$ is continuous and commutative with $T$, which also satisfies the conditions in Theorem 3.1, then we have the following consequence.

Corollary 3.1 Let $(X, F, \Delta)$ be a G-complete Menger space with $\Delta$ a continuous $t$-norm. Let $T: X \rightarrow X$ satisfying the following inequality:

$$
\frac{1}{F_{T x, T y}(\phi(c t))}-1 \leq \varphi\left\{\frac{1}{F_{x, y}(\phi(t))}-1\right\}
$$

for all $x, y \in X, c \in(0,1), \varphi \in \Phi, \phi$ is a $\phi$-function, $t>0$, such that $F_{x, y}(\phi(t))>0$. Then $T$ has a unique fixed point, i.e., there exists $u \in X$ such that $u=A u=T u$.

Remark 3.1 Corollary 3.1 is Theorem 2.1 of [16].

Theorem 3.2 Let $(X, F, \Delta)$ be a G-complete Menger space with $\Delta$ a continuous t-norm and $\Delta \leq \Delta_{p}$. Let $T: \underbrace{X \times X \times \cdots \times X}_{m \text {-times }} \rightarrow X$ and $A: X \rightarrow X$ be two mappings satisfying the following inequality:

$$
\begin{aligned}
& \frac{1}{F_{T(x, y, \ldots, z), T(p, q, \ldots, r)}(\phi(c t))}-1 \\
& \quad \leq \varphi\left\{\sqrt[m]{\Delta\left(\frac{1}{F_{A x, A p}(\phi(t))}-1, \Delta\left(\frac{1}{F_{A y, A q}(\phi(t))}-1, \ldots, \frac{1}{F_{A z, A r}(\phi(t))}-1\right)\right)}\right\}
\end{aligned}
$$


for all $x, y, \ldots, z, p, q, \ldots, r \in X, c \in(0,1), \varphi \in \Phi, \phi$ is a $\phi$-function, $t>0$, such that $F_{A x, A p}(\phi(t))>0, F_{A y, A p}(\phi(t))>0, \ldots, F_{A z, A r}(\phi(t))>0$, where $T(X \times X \times \cdots \times X) \subset A(X), A$ is continuous and commutative with $T$. Then there exists a unique multipled common fixed point of $A$ and $T$, i.e., $u \in X$ such that $u=A u=T(u, u, \ldots, u)$.

Proof Since $\Delta \leq \Delta_{p}$, we get

$$
\begin{aligned}
& \frac{1}{F_{T(x, y, \ldots, z), T(p, q, \ldots, r)(\phi(c t))}-1} \\
& \quad \leq \varphi\left\{\sqrt[m]{\Delta\left(\frac{1}{F_{A x, A p}(\phi(t))}-1, \Delta\left(\frac{1}{F_{A y, A q}(\phi(t))}-1, \ldots, \frac{1}{F_{A z, A r}(\phi(t))}-1\right)\right)}\right\} \\
& \quad \leq \varphi\left\{\sqrt[m]{\left(\frac{1}{F_{A x, A p}(\phi(t))}-1\right)\left(\frac{1}{F_{A y, A q}(\phi(t))}-1\right) \cdots\left(\frac{1}{F_{A z, A r}(\phi(t))}-1\right)}\right\} \\
& \quad \leq \varphi\left\{\frac{\left(\frac{1}{F_{A x, A p}(\phi(t))}-1\right)+\left(\frac{1}{F_{A y, A q}(\phi(t))}-1\right)+\cdots+\left(\frac{1}{F_{A z, A r}(\phi(t))}-1\right)}{m}\right\} .
\end{aligned}
$$

Then we can complete the proof by Theorem 3.1.

\section{An illustration}

Example 4.1 Let $X=[0,1], d$ be the usual metric on $X$. Define $T: \underbrace{X \times X \times \cdots \times X}_{m \text {-times }} \rightarrow X$ as $T\left(x_{1}, x_{2}, \ldots, x_{m}\right)=\frac{x_{1}+x_{2}+\cdots+x_{m}}{3 m}, A: X \rightarrow X$ as $A x=\frac{x}{2}$ and

$$
F_{x, y}(t)= \begin{cases}\frac{t}{t+d(x, y)}, & t>0, \\ 0, & t=0\end{cases}
$$

for all $x_{1}, x_{2}, \ldots, x_{m}, x, y \in X$, where $T(X \times X \times \cdots \times X) \subset A(X)$. Then $\left(X, F, \Delta_{m}\right)$ is a complete Menger PM-space, $\Delta_{m}$ is a continuous $t$-norm. Define $\varphi \in \Phi$ by $\varphi(t)=\frac{99 t}{100}$ and $\phi(t)=\frac{t}{5}$ for all $t>0, c=\frac{9}{10}$. We obtain

$$
\begin{aligned}
& \frac{1}{F_{T\left(x_{1}, x_{2}, \ldots, x_{m}\right), T\left(y_{1}, y_{2}, \ldots, y_{m}\right)}(\phi(c t))}-1=\frac{\left|T\left(x_{1}, x_{2}, \ldots, x_{m}\right)-T\left(y_{1}, y_{2}, \ldots, y_{m}\right)\right|}{\phi(c t)} \\
& =\frac{50\left|\left(x_{1}+x_{2}+\cdots+x_{m}\right)-\left(y_{1}+y_{2}+\cdots+y_{m}\right)\right|}{27 m t}, \\
& \varphi\left\{\frac{\left(\frac{1}{F_{A x_{1}, A y_{1}}(\phi(t))}-1\right)+\left(\frac{1}{F_{A x_{2}, A y_{2}}(\phi(t))}-1\right)+\cdots+\left(\frac{1}{F_{A x_{m}, A y_{m}}(\phi(t))}-1\right)}{m}\right\} \\
& =\varphi\left\{\frac{\left|A x_{1}-A y_{1}\right|+\left|A x_{2}-A y_{2}\right|+\cdots+\left|A x_{m}-A y_{m}\right|}{m \phi(t)}\right\} \\
& \quad=\frac{99\left(\left|x_{1}-y_{1}\right|+\left|x_{2}-y_{2}\right|+\cdots+\left|x_{m}-y_{m}\right|\right)}{40 m t} .
\end{aligned}
$$

It is obvious that

$$
\begin{aligned}
& \frac{1}{F_{T\left(x_{1}, x_{2}, \ldots, x_{m}\right), T\left(y_{1}, y_{2}, \ldots, y_{m}\right)}(\phi(c t))}-1 \\
& \quad \leq \varphi\left\{\frac{\left(\frac{1}{F_{A x_{1}, A y_{1}}(\phi(t))}-1\right)+\left(\frac{1}{F_{A x_{2}, A y_{2}}(\phi(t))}-1\right)+\cdots+\left(\frac{1}{F_{A x_{m}, A y_{m}}(\phi(t))}-1\right)}{m}\right\}
\end{aligned}
$$


for all $t>0$. Thus all the conditions of Theorem 3.1 are satisfied. Therefore, 0 is the unique multipled common fixed point of $A$ and $T$.

\author{
Competing interests \\ The authors declare that they have no competing interests.
}

Authors' contributions

All authors contributed equally. All authors read and approved the final manuscript.

\title{
Acknowledgements
}

The authors would like to thank the editor and the referees for their constructive comments and suggestions. The research was supported by the National Natural Science Foundation of China $(11361042,11326099,11461045,11071108)$ and the Provincial Natural Science Foundation of Jiangxi, China (20132BAB201001, 20142BAB211016, 2010GZS0147).

Received: 17 May 2015 Accepted: 23 July 2015 Published online: 07 August 2015

\section{References}

1. Menger, K: Statistical metrics. Proc. Natl. Acad. Sci. USA 28, 535-537 (1942)

2. Schweizer, B, Sklar, A: Statistical metric spaces. Pac. J. Math. 10, 313-334 (1960)

3. Zhu, CX: Several nonlinear operator problems in the Menger PN space. Nonlinear Anal. 65(7), 1281-1284 (2006)

4. Zhu, CX: Research on some problems for nonlinear operators. Nonlinear Anal. 71(10), 4568-4571 (2009)

5. Ćirić, L: Solving the Banach fixed point principle for nonlinear contractions in probabilistic metric spaces. Nonlinear Anal. 72(3), 2009-2018 (2010)

6. Chauhan, S, Pant, BD: Fixed point theorems for compatible and subsequentially continuous mappings in Menger spaces. J. Nonlinear Sci. Appl. 7(2), 78-89 (2014)

7. Xiao, JZ, Zhu, XH, Liu, XY: An alternative characterization of probabilistic Menger spaces with H-type triangular norms. Fuzzy Sets Syst. 227, 107-114 (2013)

8. $\mathrm{Wu}, \mathrm{ZQ}, \mathrm{Zhu}, \mathrm{CX}, \mathrm{Li}$, J: Common fixed point theorems for two hybrid pairs of mappings satisfying the common property (E.A) in Menger PM-spaces. Fixed Point Theory Appl. 2013(1), 25 (2013)

9. Sehgal, VM, Bharucha-Reid, AT: Fixed points of contraction mappings on probabilistic metric spaces. Theory Comput. Syst. 6(1), 97-102 (1972)

10. Khan, MS, Swaleh, M, Sessa, S: Fixed point theorems by altering distances between the points. Bull. Aust. Math. Soc 30(1), 1-9 (1984)

11. Choudhury, BS, Das, K: A new contraction principle in Menger spaces. Acta Math. Sin. Engl. Ser. 24(8), 1379-1386 (2008)

12. Jachymski, J: On probabilistic $\varphi$-contractions on Menger spaces. Nonlinear Anal. 73, 2199-2203 (2010)

13. Dutta, PN, Choudhury, BS, Das, K: Some fixed point results in Menger spaces using a control function. Surv. Math. Appl. 4, 41-52 (2009)

14. Vetro, C, Vetro, P: Common fixed points for discontinuous mappings in fuzzy metric spaces. Rend. Circ. Mat. Palermo 57(2), 295-303 (2008)

15. Gopal, D, Abbas, M, Vetro, C: Some new fixed point theorems in Menger PM-spaces with application to Volterra type integral equation. Appl. Math. Comput. 232, 955-967 (2014)

16. Kutbi, MA, Gopal, D, Vetro, C, Sintunavarat, W: Further generalization of fixed point theorems in Menger PM-spaces. Fixed Point Theory Appl. 2015(1), 32 (2015)

17. Došenovć, T, Kumam, P, Gopal, D, Patel, DK, Takači, A: On fixed point theorems involving altering distances in Menger probabilistic metric spaces. J. Inequal. Appl. 2013(1), 576 (2013)

18. Wairojjana, N, Došenović, T, Rakic, D, Gopal, D, Kumam, P: An altering distance function in fuzzy metric fixed point theorems. Fixed Point Theory Appl. 2015(1), 69 (2015)

19. Xiao, JZ, Zhu, XH, Cao, YF: Common coupled fixed point results for probabilistic $\varphi$-contractions in Menger spaces. Nonlinear Anal. 74(13), 4589-4600 (2011)

20. Sedghi, S, Altun, I, Shobe, N: Coupled fixed point theorems for contractions in fuzzy metric spaces. Nonlinear Anal. 72(3), 1298-1304 (2010)

21. Luo, T, Zhu, CX, Wu, ZQ: Tripled common fixed point theorems under probabilistic $\varphi$-contractive conditions in generalized Menger probabilistic metric spaces. Fixed Point Theory Appl. 2014(1), 158 (2014)

22. Hadžić, O, Pap, E: Fixed Point Theory in Probabilistic Metric Spaces. Kluwer Academic, Dordrecht (2001)

23. Schweizer, B, Sklar, A: Probabilistic Metric Spaces. North-Holland, Amsterdam (1983) 